(55) Pandora.

\begin{tabular}{|c|c|c|c|c|c|}
\hline 1876 & & 10 & \multicolumn{2}{|c|}{$\Delta \delta$} & Ephemeride \\
\hline Aug. 9 & 一 & 2.05 & 一 & $I " \cdot 2$ & Jahrb. 1878 \\
\hline I I & - & 2.07 & - & $5 \cdot I$ & $\gg$ \\
\hline
\end{tabular}

(89) Julia.

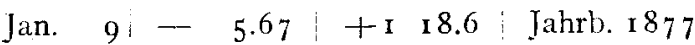
(12 I) Hermione.

Jan. 7!- $41.22+50.2 \mid$ Circul. 38 $8-41.33+47.4 \quad$ \%
$9-41.59+47.6$

(85) Jo.

1877
Mai 6 - $65.93-1$

(9i) A egina.

Mai $16 !+2.15$ - 8.8 Jahrb. 1879
(107) Camilla.

\begin{tabular}{r|c|c|c}
\hline 1877 & $\Delta \alpha$ & $\Delta \delta$ & Ephemeride \\
\hline März 17 & +0.74 & +20.3 & Circul. 67 \\
22 & +1.61 & +23.7 & ?
\end{tabular}

(Io) Hygieia.

1880

Nor. $25-8.19-32.3$ Jahrb. I 882

(78) Diana.

März I 8 - $7.8 \mathrm{I}:+1 \quad 10.4 ;$ Circul. I 3 I

(I2) Victoria.

r 88 I

März I - 5.88; + 33.8 ! Jahrb. 1883

(173) Ino.

Mai $23+0.5^{8}-11.8:$ Circul. 157 $24+1.01-15.7$ *

Beobachter waren:

$\mathrm{H}$ e $\mathrm{m}$ e r k u $\mathrm{n}$ e $\mathrm{n}$

H. B.: H. G. van de Sande Bakhuyzen; E. B.: Dr. E. F. van de Sande Bakhuyzen; K.: Prof. J. C. Kapteyn;

W.: J. H. Wilterdink.

Die Ziffern 6 und 7 deuten an, dass der Planet mit dem 6-oder 7 -zölligen Refractor beobachtel ist.

Leiden 1883 . Oct. 5 .

H. G. wan de Sande Bakhuyzen.

\title{
Beobachtung des Planeten (234)
}

angestellt von B. won Engelhardt am Fadenmicrometer des Aequatoreals (von r" engl. Oeffnung) seiner Privatsternwarte zu Dresden.

\begin{tabular}{|c|c|c|c|c|c|c|c|c|c|}
\hline $188_{3}$ & M. Z. Dresd. & $\Delta \alpha$ & $\Delta \delta$ & Gr. & $\alpha$ app. & $\log p \cdot \Delta$ & $\delta$ app. & $\log p \cdot A$ & $*$ \\
\hline Sept. 3 & $I x^{\mathrm{h}} 33^{\mathrm{m}} 37^{\mathrm{s}}$ & $+3^{\mathrm{m}} 27^{\mathrm{s}} \cdot 79$ & + 9' & 9.5 & $2 \mathrm{I}^{\mathrm{b}} \mathrm{II}^{\mathrm{m}}{ }_{1}^{\mathrm{s}}: 78$ & 9.093 & - I9 $9^{\circ} 10^{\prime} 29^{\prime \prime} 7$ & 0.914 & \\
\hline
\end{tabular}

Angenommener mittl. Ort des Vergleichsterns für r883.0.

\begin{tabular}{c|c|c|c}
\hline$*$ & $\alpha \mathrm{I} 883.0$ & $\delta \mathrm{I} 883.0$ & Autorität \\
\hline & $2 \mathrm{I}^{\mathrm{h}} 7^{\mathrm{m}} 29^{\mathrm{s}} .82$ & $-\mathrm{I}^{\circ} 20^{\prime} \mathrm{III}^{\prime \prime 2}$ & $\mathrm{AOe}_{2} 21229$
\end{tabular}

Correction der Ephemeride im Berliner Circular No. $212:+10^{\text {s }}$ 0:0.

Dresden I 883 September I 5 .

B. von Engelhardt.

\section{In halt:}

Zu. Nr. 255x, H. G. van de Sande Bakhuyzen. Beobachtungen von Planeten am 6- und am 7-zölligen Refractor der Leidentr Siternwarte. 97 B. v. Engelhardt. Beobachtung des Planeten (234). in 1 . 